PROCEEDINGS OF THE AMERICAN MATHEMATICAL SOCIETY

Volume 126, Number 3, March 1998, Pages 677-685

S $0002-9939(98) 04439-6$

\title{
ALGEBRAIC CUTS
}

\author{
DAN EDIDIN AND WILLIAM GRAHAM
}

(Communicated by Ron Donagi)

\begin{abstract}
In this note we give an algebraic version of a construction called symplectic cutting, which is due to Lerman. Our construction is valid for projective varieties defined over arbitrary fields. Using the equivariant intersection theory developed by the authors, it is a useful tool for studying quotients by torus actions. At the end of the paper, we give an algebraic proof of the Kalkman residue formula and use it to give some formulas for characteristic numbers of quotients.
\end{abstract}

\section{INTRODUCTION}

Let $X$ be a projective variety with a linearized action of an algebraic group $G$. If $X$ is smooth, and the ground field is $\mathbb{C}$, then the geometric invariant theory quotient $X / / G$ can be identified with a quotient constructed using symplectic geometry, the "reduced space" $X_{r}$. This result, due to Mumford, Guillemin and Sternberg, connects geometric invariant theory and symplectic geometry.

Suppose now that $G=T$ is a torus, which for simplicity we will take to have dimension 1 . In $[\mathrm{L}]$, Lerman introduced a construction called symplectic cutting, which constructs a manifold $X_{c}$ related to $X$, with a $T$-action, and embeds $X_{r}$ as a component of the fixed point locus $X_{c}^{T}$. The complement of $X_{r}$ in $X_{c}$ can be identified with an open submanifold of $X$, so the other components of $X_{c}^{T}$ are certain components of $X^{T}$. The components of $X_{c}^{T}$ are linked by the localization theorem in equivariant cohomology. Thus, from knowledge of $X^{T}$ one can (via the cut space) deduce results about $X_{r}$. For example, Lerman uses cutting to prove a residue formula due to Kalkman, which is closely connected to the localization theorem of Jeffrey-Kirwan-Witten ([G-K]).

The purpose of this paper is to present an algebraic version of Lerman's construction, called algebraic cutting, which is valid over arbitrary ground fields and for possibly singular schemes. This is useful in studying $X_{r}$ from the point of view of algebraic geometry, using the equivariant intersection theory developed in [E-G] in place of equivariant cohomology. For example, Lerman's proof of Kalkman's formula becomes valid for smooth schemes over arbitrary ground fields; at the end of this paper we briefly give an adaptation of that proof.

Received by the editors September 6, 1996.

1991 Mathematics Subject Classification. Primary 14D25, 14L30.

Key words and phrases. Geometric invariant theory, Chow groups.

The first author was partially supported by the NSF, and the University of Missouri Research Board.

(C)1998 American Mathematical Society 
Throughout this paper $T$ will denote a one-dimensional split torus $\mathbb{G}_{m}, X \subset$ $\mathbf{P}(V)$ a quasiprojective variety with a linearized $T$-action, and $X^{s}$ the set of stable points for this action. An element of the group $T$ will usually be denoted by $g$. All Chow groups are assumed to have rational coefficients. $A_{T}^{*}$ denotes the $T$ equivariant Chow ring of a point; it is isomorphic to the polynomial ring $\mathbb{Q}[t]$. The sign convention for $A_{T}^{*}$ is determined as follows. We model $B T$ by $(V-0) / T=\mathbf{P}^{N}$, where $V$ is an $N+1$ dimensional vector space on which $T$ acts with all weights -1 . The element $t$ represents the hyperplane class. A representation of $T$ is an equivariant vector bundle over a point, and thus has equivariant Chern classes, coming from the induced bundle over BT. A 1-dimensional representation with

weight $a$ then has equivariant first Chern class at. The Chow ring $A_{T \times T}^{*}$ will be denoted $\mathbb{Q}\left[t_{1}, t_{2}\right]$.

\section{Algebraic Cuts}

2.1. Cutting at zero. Let $v_{1}, \ldots, v_{n}$ be a basis of $V$ consisting of weight vectors, of weights $a_{1}, \ldots, a_{n} \in \mathbb{Z}$. If $x \in \mathbf{P}(V)$ is the line spanned by $\sum x_{i} v_{i}$, define (cf. [B-P]) the weights of $x$ (denoted $\Pi(x))$ to be the set of $a_{i}$ such that $x_{i}$ is nonzero. As an algebraic analogue of the fact that 0 is a regular value of the moment map, we will assume that for all $x \in X, 0 \notin \mathbf{P} i(x)$. Thus, $X$ actually lies in $\mathbf{P}(W)$, where $W$ is the subspace of $V$ spanned by weight vectors with nonzero weights. By replacing $V$ by $W$ if necessary, we will also assume that 0 is not a weight of $T$ on $V$.

By $[\mathrm{B}-\mathrm{P}]$ stability with respect to the line bundle $\mathcal{O}_{\mathbf{P}(V)}(1)$ can be expressed in terms of the weights:

$$
X^{s}=\{x \in X \mid \Pi(x) \text { contains both positive and negative weights }\} .
$$

By assumption, 0 is not a weight of $T$ on $V$, so $X^{s s}=X^{s}$. Hence if $X$ is projective then the "reduced space" $X_{r}:=X_{s} / T$ is projective. If $X$ is smooth and the ground field is $\mathbb{C}$, then $X_{r}$ corresponds to the symplectic reduction at zero.

Define

$$
X_{>0}=\{x \in X \mid \Pi(x) \text { contains a positive weight }\} .
$$

Let $T_{\Delta}$ and $A$ denote the diagonal and antidiagonal copies of $T$ in $T \times T$. Let $\left(X \times \mathbb{A}^{1}\right)^{s}$ denote the stable points of $X \times \mathbb{A}^{1}$ with respect to the action of $A$ and the line bundle $\mathcal{L}=\mathcal{O}_{\mathbf{P}(V)}(1) \otimes 1$.

Definition 1. The algebraic cut of $X$ is the quotient $X_{c}=\left(X \times \mathbb{A}^{1}\right)^{s} / A$. This scheme has a natural $T$-action, coming from the isomorphism $T \rightarrow(T \times T) / A$, $g \longmapsto(g, 1) \bmod A$.

The main results concerning this space are two theorems, which are algebraic versions of Lerman's results. The first says that $X_{c}$ is projective (if $X$ is) and is assembled out of the pieces $X_{>0}$ and $X_{r}$.

Theorem 1. (a) If $X$ is projective then $X_{c}$ is projective.

(b) There is a T-equivariant open embedding $i_{c}: X_{>0} \hookrightarrow X_{c}$ and a T-equivariant closed embedding $j_{c}: X_{r} \hookrightarrow X_{c}$ such that $X_{c}$ is a disjoint union of $X_{>0}$ and $X_{r}$. (Here $T$ acts trivially on $X_{r}$.)

(c) A neighborhood of $X_{r}$ in $X_{c}$ is isomorphic to $X^{s} \times^{T} \mathbb{A}^{1} \rightarrow X_{r}$. 
Proof. To prove (a), we will use the fact that if $Y$ is a projective variety with a linearized $G$-action, then $Y^{s s} / G$ is projective ([GIT]). Since $X \times \mathbb{A}^{1}$ is not projective, we will construct an $A$-equivariant embedding into a projective variety $Y$ with a linearized $A$-action and show that $Y^{s s}$ coincides with $\left(X \times \mathbb{A}^{1}\right)^{s}$. We do this as follows. For any integer $j$ let $\mathbb{A}_{j}^{2}$ denote $\mathbb{A}^{2}$ with weights 0 and $j$ for the action of $T$. Likewise, set $\mathbf{P}_{j}^{1}=\mathbf{P}\left(A_{j}^{2}\right)$ with the induced $T$-action. The embedding $\mathbb{A}^{1} \hookrightarrow \mathbf{P}_{1}^{1}$ given by $z \mapsto[1: z]$ is $T$-equivariant and induces a $T \times T$-invariant embedding $p: X \times \mathbb{A}^{1} \hookrightarrow X \times \mathbf{P}_{1}^{1}$.

Let $a_{0}, \ldots, a_{n}$ be the weights of $T$ on $X$. Fix an integer $N$ which is greater than any $a_{i}$. There is a product action of $T \times T$ on $V \otimes \mathbb{A}_{N}^{2}$. The weights $T_{\Delta}$ on $V \otimes \mathbb{A}_{N}^{2}$ are $a_{0}, \ldots, a_{n}, a_{0}+N, \ldots, a_{n}+N$, and the weights of $A$ are $a_{0}, \ldots, a_{n}, a_{0}-$ $N, \ldots, a_{n}-N$. Since $N$ is greater than each $a_{i}, 0$ is not a weight of $A$ on $V \otimes \mathbb{A}_{N}^{2}$. Thus, by [B-P] all semi-stable points (with respect to $A$ ) are stable.

Consider the $T \times T$-equivariant finite morphism $\psi: X \times \mathbf{P}_{1}^{1} \rightarrow \mathbf{P}\left(V \otimes \mathbb{A}_{N}^{2}\right)$ given by

$$
\left(\left[x_{0}: \cdots: x_{n}\right],\left[z_{0}: z_{1}\right]\right) \mapsto\left[x_{0} z_{0}^{N}: \cdots: x_{n} z_{0}^{N}: x_{0} z_{1}^{N}: \cdots x_{n} z_{1}^{N}\right] .
$$

Let $\mathcal{M}=\psi^{*} \mathcal{O}(1)$.

Let $\mathbf{P}\left(V \otimes \mathbb{A}_{N}^{2}\right)^{s}$ denote the stable points for the action of $A$ with respect to $\mathcal{O}(1)$ and $\left(X \times \mathbf{P}_{1}^{1}\right)^{s}$ denote the stable points for the action of $A$ with respect to $\mathcal{M}$. Since $\psi$ is finite and $X \times \mathbf{P}_{1}^{1}$ is proper, by [GIT, Theorem 1.19], $\left(X \times \mathbf{P}_{1}^{1}\right)^{s}=$ $\psi^{-1}\left(\mathbf{P}\left(V \otimes \mathbb{A}_{N}^{2}\right)^{s}\right)$. Since 0 is not a weight, all semi-stable points are stable.

The stable points in $\mathbf{P}\left(V \otimes \mathbb{A}_{N}^{2}\right)$ are described in [B-P]: if we let $\Pi_{A}(y)$ denote the $A$-weights of $y \in \mathbf{P}\left(V \otimes \mathbb{A}_{N}^{2}\right)^{s}$, then $y$ is stable if and only if $\Pi_{A}(y)$ contains both positive and negative weights. Hence the unstable points $(x, z)$ of $X \times \mathbf{P}_{1}^{1}$ with respect to $\mathcal{M}$ are of 3 types:

(i) $z=[0: 1], x$ arbitrary;

(ii) $z=[1: 0], \Pi(x)$ all positive or all negative;

(iii) $z \neq[0: 1],[1: 0], \Pi(x)$ all negative.

In particular, the stable points of $X \times \mathbf{P}_{1}^{1}$ lie in the open set $X \times \mathbb{A}^{1} \subset X \times \mathbf{P}^{1}$. Restricting to points $(x, w)$ in the open set $X \times \mathbb{A}^{1}$, we see that there are two types of unstable points:

$\left(i i^{\prime}\right) w=0, \Pi(x)$ all positive or all negative;

$\left(i i i^{\prime}\right) w \neq 0, \Pi(x)$ all negative.

The restriction of $\mathcal{M}$ to $X \times \mathbb{A}^{1}$ is $\mathcal{L}$. As in Definition 1 , let $\left(X \times \mathbb{A}^{1}\right)^{s}$ denote the set of stable points relative to to $T$ and $\mathcal{L}$. By [GIT, Prop. 1.18] the inclusion $p: X \times \mathbb{A}^{1} \subset X \times \mathbf{P}_{1}^{1}$ induces a reverse inclusion

$$
\left(X \times \mathbb{A}^{1}\right)^{s} \supset p^{-1}\left(X \times \mathbf{P}_{1}^{1}\right)^{s}=\left(X \times \mathbf{P}_{1}^{1}\right)^{s} \cap\left(X \times \mathbb{A}^{1}\right) .
$$

However, since $\left(X \times \mathbf{P}_{1}^{1}\right)^{s} \subset X \times \mathbb{A}^{1}$ this implies

$$
\left(X \times \mathbb{A}^{1}\right)^{s} \supset\left(X \times \mathbf{P}_{1}^{1}\right)^{s} .
$$

To prove that $X_{c}=\left(X \times \mathbb{A}^{1}\right)^{s} / A$ is projective it suffices to show that this inclusion is an equality, since the quotient $\left(X \times \mathbf{P}_{1}^{1}\right)^{s} / T$ is projective. In other words, we must show that all points of types $\left(\mathrm{ii}^{\prime}\right)$ and $\left(\mathrm{iii}^{\prime}\right)$ are $\mathcal{M}$-unstable. We will do this the old fashioned way, and show that any $A$-invariant element of $H^{0}\left(X \times \mathbb{A}^{1}, \mathcal{L}^{d}\right)$ vanishes on points of types (ii') and (iii'). In doing so, we may assume $d>>0$.

For $d>>0$, the restriction map

$$
H^{0}(\mathbf{P}(V), \mathcal{O}(d)) \cong S^{d}\left(V^{*}\right) \rightarrow H^{0}(X, \mathcal{O}(d)),
$$


is surjective (here $V^{*}$ is the dual vector space to $V$ ). If $\xi \in V^{*}$ is a positive (resp. negative) weight vector and if $x \in X$ has all positive (resp. negative) weights, then $\xi$ (viewed as a section of $\mathcal{O}(1)$ ) vanishes at $x$.

Now if $s \in S^{d}\left(V^{*}\right)$ is a $T$-invariant section, then it is a sum of monomials in weight vectors, with each monomial containing both positive and negative weight vectors. Thus, if $s \in S^{d}\left(V^{*}\right)^{T}$ and $\Pi(x)$ consists of all positive or all negative weights, then $s(x)=0$. Similarly, if $s \in S^{d}\left(V^{*}\right)$ is a sum of monomials and each monomial contains a negative weight vector (in particular if the weight of $s$ is negative), then $s(x)=0$ if $\Pi(x)$ has only negative weights.

Now if $s \in H^{0}(X, \mathcal{O}(d))$ has $T$-weight $k$, and $f_{l}(w)=w^{l} \in \mathcal{O}\left(\mathbb{A}^{1}\right)$, then $s \otimes f_{l}$ has weight $k+l$ for the anti-diagonal torus $A$ (note that $f_{l}$ has $T$-weight $-l$ ). Thus, a general $A$-invariant section $\tau$ of $H^{0}\left(X \times \mathbb{A}^{1}, \mathcal{L}^{d}\right)$ is of the form $\tau=\sum \tau_{l} f_{l}$, where $\tau_{l} \in H^{0}(X, \mathcal{O}(d))$ has $T$-weight $-l$.

We want to show that if $\tau$ is $A$-invariant then $\tau$ vanishes on $(x, w)$ of types $\left(i i^{\prime}\right)$ and $\left(i i i^{\prime}\right)$. On type $\left(i i^{\prime}\right)$, we have

$$
\tau(x, 0)=\sum \tau_{l}(x) f_{l}(0)=\tau_{0}(x) .
$$

But $\tau_{0}$ is $T$-invariant, and $(x, 0)$ of type $\left(i i^{\prime}\right)$ means that $\Pi(x)$ consists of all positive or all negative weights, so by the above remarks, $\tau_{0}(x)=0$. If $(x, w)$ is of type $\left(i i i^{\prime}\right)$, then $\Pi(x)$ consists of all negative weights; since the weight of $\tau_{i}=-i \leq 0$, we have $\tau_{i}(x)=0$ for all $i$. Hence $\tau$ vanishes on $(x, w)$ of types $\left(i i^{\prime}\right)$ and $\left(i i i^{\prime}\right)$, as desired. This proves (a).

We now prove (b) and (c). The embedding $i_{c}$ is defined to be the quotient by $A$ of the open embedding

$$
X_{>0} \times T \hookrightarrow\left(X \times \mathbb{A}^{1}\right)^{s} .
$$

The embedding $j_{c}$ is defined to be the quotient by $A$ of the embedding

$$
J: X^{s} \times\{0\} \hookrightarrow\left(X \times \mathbb{A}^{1}\right)^{s} ;
$$

$J$ is closed since, from the description of $\left(X \times \mathbb{A}^{1}\right)^{s}$ given in the proof of (a), we have $\left(X \times \mathbb{A}^{1}\right)^{s} \cap X \times\{0\}=X^{s} \times\{0\}$. The embedding $i_{c}$ (resp. $j_{c}$ ) is open (resp. closed) since it is the quotient of an open (resp. closed) embedding. This proves (b).

A neighborhood of $X^{s} \times\{0\}$ in $\left(X \times \mathbb{A}^{1}\right)^{s}$ is given by $X^{s} \times \mathbb{A}^{1}$. It follows that a neighborhood of $X_{r}$ in $X_{c}$ is $T$-equivariantly isomorphic to the fibration

$$
\left(X^{s} \times \mathbb{A}^{1}\right) / A \rightarrow\left(X^{s} \times\{0\}\right) / A .
$$

This is isomorphic to the fibration

$$
X^{s} \times^{T} \mathbb{A}^{1} \rightarrow X_{r},
$$

proving (c).

Remark. In general $T$ will act with finite stabilizers on $X^{s}$. If $T$ acts freely on $X^{s}$, then $X_{r}$ is regularly embedded with normal bundle $X^{s} \times{ }^{T} \mathbb{A}^{1} \rightarrow X_{r}$. We will see in Theorem 2 that even if the action of $T$ is not free, there is an equivariant pullback and self-intersection formula. 
2.2. Cutting at any point. Instead of considering stable points with respect to the invertible sheaf $\mathcal{O}_{\mathbf{P}(V)}(1)$ we can consider stable points with respect to other linearizations. Let $q=a / n$ be a rational number which is not a weight of $\Pi(x)$ for any $x \in X$ (we no longer require that 0 is not a weight). Then we define

$$
X^{s}(q)=\{x \in X \mid \Pi(x) \text { contains both weights }>q \text { and weights }<q\} .
$$

Define a $T$-action on $S^{n} V$ by $g \cdot v^{n}=g^{-a}(g \cdot v)^{n}$. Embed $\mathbf{P}(V)$ in $\mathbf{P}\left(S^{n} V\right)$ by the Veronese embedding, and let $\mathcal{M}(q)$ denote the pullback to $X$ of $\mathcal{O}_{\mathbf{P}\left(S^{n} V\right)}(1)$. Then ([B-P, 1.2]) $X^{s}(q)$ is the set of stable points of $X$ with respect to $\mathcal{M}(q)$. The quotient $X_{r}(q)=X^{s}(q) / T$ corresponds (in the symplectic picture) to the reduction of $X$ at $q$.

Likewise we can define (in the obvious fashion) the open subscheme $X_{>q}$ of $X$. We let $\mathcal{L}(q)$ denote the line bundle $\mathcal{M}(q) \otimes 1$ on $X \times \mathbb{A}^{1}$, and define $X_{c}(q)=$ $\left(X \times \mathbb{A}^{1}\right)^{s} / A$, where now stability is defined with respect to $\mathcal{L}(q)$. The analogue of Theorem 1 holds for $X_{c}(q)$. This can be deduced from Theorem 1 by embedding $X$ into $\mathbf{P}\left(S^{n} V\right)$ as above.

\section{Equivariant interSECTION THEORY AND ALGEBRAIC CUTS}

3.1. Review of equivariant Chow groups. In this section we recall the definition and some of the basic properties of equivariant Chow groups [E-G]. For simplicity we will assume that all schemes are quasiprojective and that any group actions are linearized.

Let $G$ be a $g$-dimensional group, $X$ an $n$-dimensional scheme and $V$ a representation of $G$ of dimension $l$. Assume that there is an open set $U \subset V$ such that a principal bundle quotient $U \rightarrow U / G$ exists, and that $V-U$ has codimension more than $i$. Let $X_{G}=(X \times U) / G$.

Definition 2. Set $A_{i}^{G}(X)=A_{i+l-g}\left(X_{G}\right)$, where $A_{*}$ is the usual Chow group. This definition is independent of the choice of $V$ and $U$ as long as $V-U$ has sufficiently high codimension.

Remark. Because $X \times U \rightarrow X \times{ }^{G} U$ is a principal $G$-bundle, cycles on $X \times{ }^{G} U$ exactly correspond to $G$-invariant cycles on $X \times U$. Since we only consider cycles of codimension smaller than the dimension of $X \times(V-U)$, we may in fact view these as $G$-invariant cycles on $X \times V$. Thus every class in $A_{i}^{G}(X)$ is represented by a cycle in $Z_{i+l}(X \times V)^{G}$, where $Z_{*}(X \times V)^{G}$ indicates the group of cycles generated by invariant subvarieties. Conversely, any cycle in $Z_{i+l}(X \times V)^{G}$ determines an equivariant class in $A_{i}^{G}(X)$.

The properties of equivariant intersection theory include the following.

(1) Functoriality for equivariant maps: proper pushforward, flat pullback, l.c.i. pullback, etc.

(2) Chern classes of equivariant bundles operate on equivariant Chow groups.

(3) If $X$ is smooth, then $\bigoplus A_{*}^{G}(X)$ has a ring structure. (This follows from (1), since the diagonal $X \hookrightarrow X \times X$ is an equivariant regular embedding when $X$ is smooth.)

(4) There is a localization theorem for torus actions.

Lemma 1. Let $G$ be a group and $X$ a quasi-projective $G$-scheme. Suppose that $X$ is the set of stable points for the linear action of a normal (reductive) subgroup $H \subset G$. Then $A_{i}^{G}(X)=A_{i-h}^{G / H}(X / H)$, where $h=\operatorname{dim} H$. 
Proof. Let $V$ be a representation of $G / H$, and let $U$ be an open set on which $G / H$ acts freely. Then $G$ acts on $X \times U$ with finite stabilizers. Thus, by [E-G, Theorem 3] $A_{*}^{G}(X \times U)=A_{*}((X \times U) / G)$. Now $(X \times U) / G=G / H(X \times U) / H$. Since $H$ acts trvially on $U,(X \times U) / H=X / H \times U$. Thus $(X \times U) / G=X / H \times{ }^{G / H} U$.

Hence if $V-U$ has sufficiently high codimension then $A_{*}(X \times U)=A_{*}^{G / H}(X / H)$. Thus, the lemma follows from the homotopy property of equivariant Chow groups.

Lemma 2. Suppose $X$ is a $G$-scheme, $Y$ is an $H$-scheme, and $Q \subset X \times Y$ is $G \times H$-invariant and flat over $X$. Let $\pi: Q \rightarrow X$ be the projection. Then there is a pullback

$$
\pi^{*}: A_{*}^{G}(X) \rightarrow A_{*}^{G \times H}(Q) .
$$

Proof. Let $V_{G}$ be a representation of $G$. Let $p_{Q}: Q \times V_{G} \rightarrow X \times V_{G}$ be the projection onto the first and third factors. If $Z \subset\left(X \times V_{G}\right)$ set $\pi_{Q}^{*}[Z]=\left[\pi_{Q}^{-1} Z\right]$. This is an invariant cycle in $Q \times V_{G}$ and so defines an element of $A_{*}^{G \times H}(Q)$. The usual arguments of equivariant theory show that this is well defined.

Theorem 2. Let $X$ be a quasiprojective scheme with a linearized T-action and cut scheme $X_{c}=X_{c}(q)$ for some $q \in \mathbb{Q}$. Then:

(a) There is a pullback of rational equivariant Chow groups

$$
j_{c}^{*}: A_{*}^{T}\left(X_{c}\right) \rightarrow A_{*}^{T}\left(X_{r}\right)
$$

such that $j_{c *} j_{c}^{*}$ is multiplication by $t$ under the map $A_{T}^{1} \rightarrow A_{T}^{1}\left(X^{s}\right) \cong A^{1}\left(X_{r}\right)$.

(b) There is a map $s: A_{*}^{T}(X) \rightarrow A_{*}^{T}\left(X_{c}\right)$ such that $i_{c}^{*} \circ s=i^{*}$. Here $i$ and $i_{c}$ denote the embeddings of $X_{>0}$ into $X$ and $X_{c}$, respectively.

(c) If $F \subset X_{>0}^{T}$, let $i_{F}$ and $i_{F, c}$ denote the embeddings of $F$ into $X$ and $X_{c}$ respectively. Then $i_{F, c}^{*} \circ s=i_{F}^{*}$.

$(d)$ As maps $A_{*}^{T}(X) \rightarrow A_{*}\left(X_{r}\right)$, we have $\mathcal{F} \circ j_{c}^{*} \circ s=r^{*}$, where $\mathcal{F}$ denotes the forgetful map from equivariant Chow groups to ordinary Chow groups, and $r^{*}$ is the restriction $A_{*}^{T}(X) \rightarrow A_{*}^{T}\left(X_{s}\right) \cong A_{*}\left(X_{r}\right)$.

Remark. Because $T$ acts trivially on $X_{r}$, we have $A_{T}^{*}\left(X_{r}\right) \cong A_{*}\left(X_{r}\right) \otimes_{\mathbb{Q}} A_{*}^{T}$. Thus, if $\alpha \in A_{*}^{T}(X)$, we can write $j_{c}^{*} \circ s(\alpha)=\sum \beta_{i} t^{i}$. The content of (d) is that $\beta_{0}=r(\alpha)$.

Proof. To simplify notation we assume $q=0$. By the discussion of Section 2.2 this implies the theorem for $q \neq 0$ as well.

(a) The $T \times T$-equivariant regular embedding $J: X^{s} \times\{0\} \hookrightarrow\left(X \times \mathbb{A}^{1}\right)^{s}$ gives a pullback $J^{*}$ on $T \times T$-equivariant Chow groups. As noted in the proof of Theorem 1 , the map $j_{c}$ is the quotient of the map $J$ by $A$. We have an isomorphism $T \rightarrow$ $(T \times T) / A, g \mapsto(g, 1)=(1, g) \bmod A$. By Lemma 1 we can identify

$$
A_{*}^{T}\left(X_{c}\right)=A_{*}^{T \times T}\left(\left(X \times \mathbb{A}^{1}\right)^{s}\right), A_{*}^{T}\left(X_{r}\right)=A_{*}^{T \times T}\left(X^{s} \times\{0\}\right) .
$$

Let $j_{c}^{*}$ correspond to $J^{*}$ under this identification. The $T \times T$-equivariant normal bundle to $X^{s} \times\{0\}$ in $\left(X \times \mathbb{A}^{1}\right)^{s}$ is the trivial line bundle, where $T \times T$ acts with weight $(0,1)$. Thus $J_{*} J^{*}$ is multiplication by $t_{2} \in A_{T \times T}^{*}$. With our identifications, multiplication by either $t_{1}$ or $t_{2}$ in $A_{T \times T}^{*}=\mathbb{Q}\left[t_{1}, t_{2}\right]$ corresponds to multiplication by $t \in A_{T}^{*}=\mathbb{Q}[t]$. Hence $j_{c *} j_{c}^{*}$ is multiplication by $t$. This proves (a).

(b) We have inclusions

$$
X_{>0} \times T \stackrel{I}{\rightarrow}\left(X \times \mathbb{A}^{1}\right)^{s} \stackrel{k}{\rightarrow} X \times \mathbb{A}^{1} .
$$


The map $i_{c}$ is the quotient of $I$ by $A$. Consider the following commutative diagram:

$$
\begin{array}{cccc}
A_{*}^{T}(X) \stackrel{\pi^{*}}{\rightarrow} A_{*}^{T \times T}\left(X \times \mathbb{A}^{1}\right) \stackrel{k^{*}}{\rightarrow} & A_{*}^{T \times T}\left(\left(X \times \mathbb{A}^{1}\right)^{s}\right) & \stackrel{I^{*}}{\rightarrow} & A_{*}^{T \times T}\left(X_{>0} \times T\right) \\
f \downarrow & & g \downarrow \\
A_{*}^{T}\left(X_{c}\right) & \stackrel{i_{c}^{*}}{\longrightarrow} & A_{*}^{T}\left(X_{>0}\right)
\end{array}
$$

The vertical maps are from Lemma 1 ; here $G=T \times T, H=A$, and $G / H=$ $(T \times T) / A \cong T$. We define

$$
s: A_{*}^{T}(X) \rightarrow A_{*}^{T}\left(X_{c}\right)
$$

to be $s=f k^{*} \pi^{*}$. If $Z$ is a subvariety of $X$, then

$$
i_{c}^{*} \circ s([Z])=g I^{*} k^{*} \pi^{*}[Z]=g\left[\left(Z \cap X_{>0}\right) \times T\right] .
$$

Since $A$ acts freely on $X_{>0} \times T$,

$$
g\left[\left(Z \cap X_{>0}\right) \times T\right]=\left[Z \cap X_{>0}\right]=i^{*}[Z],
$$

proving (b). Part (c) follows from (b).

The proof of (d) is similar to that of (b).

3.2. Kalkman's residue formula. Following [L], we use algebraic cutting to prove the residue formula of Kalkman.

Theorem 3. Let $X \subset \mathbf{P}(V)$ be a smooth $n$-dimensional projective variety with a linearized T-action. Fix a rational $q$ which is not a weight of $x \in X$. Let $r^{*}: A_{T}^{*}(X) \rightarrow A^{*}\left(X_{r}\right)$ be as defined above, and let $\alpha \in A_{T}^{n-1}(X)$. Then

$$
\operatorname{deg}\left(r^{*}(\alpha) \cap\left[X_{r}\right]\right)=-\sum \operatorname{deg}\left(\left(\operatorname{Res}_{t=0} \frac{i_{F}^{*} \alpha}{c_{d_{F}}^{T}\left(N_{F} X\right)}\right) \cap[F]_{T}\right),
$$

where the sum is over the components $F$ of $X_{>q}^{T}$, and $d_{F}$ is the codimension of $F$.

Proof. To simplify notation assume that $q=0$ in the proof. Recall that $X_{c}^{T}=$ $X_{>0}^{T} \cup X_{r}$. Each $F \subset X_{>0}^{T}$ is regularly embedded in $X_{c}$, so the self-intersection formula applies. Although $X_{r}$ need not be regularly embedded in $X_{c}$, a selfintersection formula still holds (Theorem 2(a)). Thus the localization theorem for equivariant Chow groups [E-G2] can be applied just as if $X_{r}$ were regularly embedded, so

$$
s(\alpha) \cap\left[X_{c}\right]_{T}=\sum_{F \subset X_{>0}} i_{F, c *}\left(\frac{i_{F, c}^{*} s(\alpha)}{c_{d_{F}}^{T}\left(N_{F} X\right)} \cap[F]_{T}\right)+j_{c *}\left(\frac{j_{c}^{*} s(\alpha)}{t} \cap\left[X_{r}\right]_{T}\right) .
$$

This equality holds in $A_{*}^{T}\left(X_{c}\right) \otimes_{A_{T}^{*}} \mathcal{Q}$, where $\mathcal{Q}=\mathbb{Q}\left[t, t^{-1}\right]$. Applying Theorem $2(\mathrm{c})$, we can rewrite this as

$$
s(\alpha) \cap\left[X_{c}\right]_{T}=\sum_{F \subset X_{>0}^{T}} i_{F, c *}\left(\frac{i_{F}^{*} \alpha}{c_{d_{F}}^{T}\left(N_{F} X\right)} \cap[F]_{T}\right)+j_{c *}\left(\frac{j_{c}^{*} s(\alpha)}{t} \cap\left[X_{r}\right]_{T}\right) .
$$

Let $\pi_{Y}$ denote the projection of a proper scheme $Y$ to a point and $\pi_{Y *}$ the induced map of equivariant Chow groups. If we apply $\pi_{X_{c} *}$ to both sides of (2), the left side is zero since $\alpha$ has degree $n-1$ and the dimension of $X$ is $n$. Therefore

$$
\sum_{F \subset X_{>0}^{T}} \pi_{F *}\left(\frac{i_{F}^{*} \alpha}{c_{d_{F}}^{T}\left(N_{F} X\right)} \cap[F]_{T}\right)=-\pi_{X_{r} *}\left(\frac{j_{c}^{*} s(\alpha)}{t} \cap\left[X_{r}\right]_{T}\right) .
$$


Now, $T$ acts trivially on $F$, so $A_{T}^{*}(F)=A^{*} F \otimes_{\mathbb{Q}} A_{T}^{*}$ and $[F]_{T}=[F] \otimes 1$. The class

$$
\frac{i_{F}^{*} \alpha}{c_{d_{F}}^{T}\left(N_{F} X\right)} \in A_{T}^{*}(F) \otimes_{\mathcal{Q}} A_{T}^{*}=A^{*} F \otimes_{\mathbb{Q}} \mathcal{Q}
$$

has degree $n-1-d_{F}=\operatorname{dim} F-1$, so we can write it as $\sum \beta_{i} t^{i}$, where $\beta_{i} \in A^{*} F$ has degree $\operatorname{dim} F-1-i$. Thus

$$
\frac{i_{F}^{*} \alpha}{c_{d_{F}}^{T}\left(N_{F} X\right)} \cap[F]_{T}=\left(\sum \beta_{i} \cap[F]\right) t^{i} .
$$

When we apply $\pi_{F *}$ to this the only term that survives is the term with $t^{-1}$. A similar argument applies to the right side of (3). We can therefore rewrite (3) as

$$
\sum_{F \subset X_{>0}^{T}} \operatorname{deg}\left(\left(\operatorname{Res}_{t=0} \frac{i_{F}^{*} \alpha}{c_{d_{F}}^{T}\left(N_{F} X\right)}\right) \cap[F]\right)=\operatorname{deg} \operatorname{Res}_{t=0} \frac{j^{*} s(\alpha) \cap\left[X_{r}\right]_{T}}{t} .
$$

By the remark after Theorem 2, the right side of this equation is $r^{*}(\alpha) \cap\left[X_{r}\right]$.

3.3. Characteristic numbers of quotients. Kalkman's residue formula has a number of applications; for instance it can be used to prove the localization theorem of Jeffrey-Kirwan-Witten [G-K]. Another use of the residue formula is to compute some characteristic numbers of quotients of a smooth variety $X$ by a torus. We present some formulas when $T$ acts freely on $X^{s}(q)$ and the fixed points of $X_{>q}$ are isolated.

Let $Y=X^{s} / T$ and let $\pi: X^{s} \rightarrow Y$ be the quotient map. Since $T$ acts freely, $Y$ is smooth of dimension $n-1$ and we will use the residue formula to compute the Euler characteristic $\chi(Y)=\operatorname{deg}\left(c_{n-1}\left(T_{Y}\right)\right)$. Since the fiber of $\pi$ is $T, T_{X^{s} / Y}$ is the trivial bundle of rank 1 . There is an exact sequence of equivariant bundles on $X^{s}$

$$
0 \rightarrow \mathbf{1} \rightarrow T_{X^{s}} \rightarrow \pi^{*} T_{Y} \rightarrow 0 .
$$

Hence $r\left(c_{i}\left(T_{X^{s}}\right)\right)=c_{i}\left(T_{Y}\right)$. Since $T_{X^{s}}$ is just the restriction of $T_{X}$ to $X^{s}$, we can apply the residue formula. If $p \in X_{>q}^{T}$ is a fixed point, let $\alpha_{1}(p), \ldots, \alpha_{n}(p)$ be the weights for the $T$-action on $T_{p} X$. Then

$$
c_{n-1}^{T}\left(\left.T_{X}\right|_{p}\right)=t^{n-1} \sum_{i=1}^{n} \alpha_{1}(p) \alpha_{2}(p) \ldots \widehat{\alpha_{i}(p)} \ldots \alpha_{n}(p)
$$

and $c_{n}^{T}\left(N_{p} X\right)=t^{n} \prod_{i=1}^{n} \alpha_{i}(p)$. Thus, applying the residue formula, we have

$$
\chi(Y)=-\sum_{p \in X_{>q}^{T}} \sum_{i=1}^{n} \frac{1}{\alpha_{i}(p)} .
$$

In a similar manner we can calculate the Todd genus $\chi\left(\mathcal{O}_{Y}\right)$. By HirzebruchRiemann-Roch, $\chi\left(\mathcal{O}_{Y}\right)=\operatorname{deg}\left(\operatorname{Td}\left(T_{Y}\right)\right)$. Using the residue formula to calculate $\operatorname{deg}\left(\operatorname{Td}\left(T_{Y}\right)\right)$, we obtain

$$
\chi\left(\mathcal{O}_{Y}\right)=-\sum_{p \in X_{>q}^{T}} \operatorname{res}_{t=0}\left(\frac{1}{\prod_{i=1}^{n}\left(1-e^{-t \alpha_{i}(p)}\right)}\right)=-\sum_{p \in X_{>q}^{T}}\left(\sum_{1 \leq i \neq l \leq n} \frac{\alpha_{l}(p)}{2 \alpha_{i}(p)}\right) .
$$




\section{REFERENCES}

[B-P] M. Brion, C. Procesi, Action d'un tore dans une variété projective, in Operator algebras, unitary representations, and invariant theory (Paris 1989), Prog. in Math. 92 (1990), 509-539. MR 92m:14061

[E-G] D. Edidin, W. Graham, Equivariant intersection theory, Inventiones Math., to appear, alg-geo 9609018.

[E-G2] D. Edidin, W. Graham, Localization in equivariant intersection theory and the Bott residue formula, preprint alg-geo 9508001.

[G-K] V. Guillemin, J. Kalkman, A new proof of the Jeffrey-Kirwan localization theorem, preprint (1994)

[GIT] D. Mumford, J. Fogarty, F. Kirwan, Geometric Invariant Theory, 3rd enlarged edition, Springer-Verlag (1994). MR 95m:14012

[L] E. Lerman, Symplectic cuts, Math. Res. Letters, 2 (1995), 247-258. MR 96f:58062

Department of Mathematics, University of Missouri, Columbia Missouri 65211

E-mail address: edidin@cantor.math.missouri.edu

School of Mathematics, Institute for Advanced Study, Princeton, New Jersey 08540 Current address: Department of Mathematics, University of Georgia, Athens, Georgia 30602 